\title{
Corrigendum: Akkermansia
} muciniphila as a Model Case for the Development of an Improved Quantitative RPA Microbiome Assay

\author{
Heather J. Goux ${ }^{1}$, Dimple Chavan ${ }^{1}$, Mary Crum ${ }^{2}$, Katerina Kourentzi ${ }^{2}$ and \\ Richard C. Willson ${ }^{1,2,3 *}$ \\ ${ }^{1}$ Department of Biology and Biochemistry, University of Houston, Houston, TX, United States, ${ }^{2}$ Department of Chemical \\ and Biomolecular Engineering, University of Houston, Houston, TX, United States, ${ }^{3}$ Tecnológico de Monterrey-ITESM \\ Campus Monterrey, Monterrey, Mexico
}

Keywords: RPA, gut microbiome, Akkermansia muciniphila, bacterial quantification, point-of-need

\section{A Corrigendum on}

OPEN ACCESS

Edited by:

Pascale Alard,

University of Louisville, United States

Reviewed by:

Noora Ottman

Karolinska Institute, Sweden

*Correspondence:

Richard C. Willson

willson@uh.edu

Specialty section:

This article was submitted to Microbiome in Health and Disease,

a section of the journal

Frontiers in Cellular and Infection

Microbiology

Received: 05 November 2018

Accepted: 01 February 2019

Published: 21 March 2019

Citation:

Goux HJ, Chavan D, Crum M, Kourentzi K and Willson RC (2019)

Corrigendum: Akkermansia muciniphila as a Model Case for the

Development of an Improved Quantitative RPA Microbiome Assay. Front. Cell. Infect. Microbiol. 9:32. doi: 10.3389/fcimb.2019.00032
Akkermansia muciniphila as a Model Case for the Development of an Improved Quantitative RPA Microbiome Assay

by Goux, H. J., Chavan, D., Crum, M., Kourentzi, K., and Willson, R. C. (2018). Front. Cell. Infect. Microbiol. 8:237. doi: 10.3389/fcimb.2018.00237

In the original article, there was an error. The reported relative A. muciniphila abundance measurements failed to sufficiently take into account that variable numbers of copies of the $16 \mathrm{~S}$ rRNA gene can occur in a bacterial genome. The E. coli or A. muciniphila gDNA copies per mass of isolated DNA were determined from the threshold time (RPA) or cycle (PCR) and the appropriate standard curves. After this step, the resulting gDNA copies were not multiplied by the number of $16 \mathrm{~S}$ rRNA genes that occur per genome of the standard. The number of A. muciniphila gDNA copies should have been multiplied by three, and the number of E. coli gDNA copies should have been multiplied by seven. Only then can the relative A. muciniphila abundance be calculated as the ratio of $A$. muciniphila $16 \mathrm{~S}$ gene copies to the number of bacterial $16 \mathrm{~S}$ copies.

A correction has been made to Materials and Methods, section Determining Total Bacterial and A. muciniphila Abundance. The following paragraph has been added:

For both A. muciniphila (ATCC BAA-835) and E. coli (ATCC 35218) gDNA standards, the 16S rRNA gene sequences from the GenBank (accession nos. NR_074436.1 and EF436579, respectively) were aligned to the complete genome sequences of A. muciniphila (accession no. NC_010655.1) and E. coli (accession NZ_KK583188.1) using the NCBI BLAST. This resulted in three and seven matches (100\% in identity and composition) for A. muciniphila and E. coli, respectively. Thus, to calculate the 16S rRNA gene copies for each standard, the genomic DNA copies were multiplied by the number of 16rRNA gene copies per genome ( 3 for A. muciniphila and 7 for $E$. coli).

A correction has been made to Results, section Development of the A. muciniphila Assay, subsection Absolute A. muciniphila Abundance, paragraph three. The updated paragraph reads as follows:

The absolute A. muciniphila load of the fecal sample was estimated based on the qPCR semilogarithmic regression line as $8.91 \times 10^{4}$ gDNA copies per reaction, or $1.78 \times 10^{5}$ gDNA copies 
per $15 \mathrm{ng}$ of isolated gDNA (Figure S5), quite similar to the absolute $A$. muciniphila load of the fecal sample as determined using RPA $\left(2.99 \times 10^{5}\right.$ bacterial gDNA copies per $15 \mathrm{ng}$ of isolated gDNA).

Corrections have also been made to Results, section Development of the A. muciniphila Assay, subsection Relative A. muciniphila Abundance, paragraphs one and three. The updated paragraph one reads as follows:

Relative A. muciniphila abundance was calculated as the ratio of A. muciniphila $16 \mathrm{~S}$ copies to total bacterial 16S copies using both qPCR (using primer sets 2 and 4) and RPA (using primer sets 1 and 3 ) to show a relative abundance of 1.36 and $1.29 \%$, respectively. The relative A. muciniphila abundance of the fecal sample was determined using RPA from $3 \times 2.99 \times 10^{5}$ A. muciniphila $16 \mathrm{~S}$ copies per $15 \mathrm{ng}$ of gDNA divided by $7 \times 1.01 \times 10^{7}$ bacterial 16 S copies per $15 \mathrm{ng}$ of gDNA) and from PCR $5.34 \times 10^{5}$ A. muciniphila $16 \mathrm{~S}$ copies $/ 3.91 \times 10^{7}$ bacterial $16 \mathrm{~S}$ copies) as $3 \times 1.78 \times 10^{5}$ A. muciniphila $16 \mathrm{~S}$ copies per $15 \mathrm{ng}$ of gDNA divided by $7 \times 5.58 \times 10^{6}$ bacterial $16 \mathrm{~S}$ copies per 15 ng of gDNA.
The updated paragraph three reads as follows:

When compared to sequencing, RPA gave a slightly lower relative A. muciniphila abundance in the fecal sample. This result could have been due to off-target amplification of the bacteria-specific primers. Note that the accuracy of the relative A. muciniphila abundance RPA assay, as with all nucleic acidbased assays, is highly dependent upon the quality of the primers. RPA assay sensitivity could perhaps be improved by increasing the primer specificity.

The authors apologize for these errors and state that this does not change the scientific conclusions of the article in any way. The original article has been updated.

Copyright (c) 2019 Goux, Chavan, Crum, Kourentzi and Willson. This is an openaccess article distributed under the terms of the Creative Commons Attribution License (CC BY). The use, distribution or reproduction in other forums is permitted, provided the original author(s) and the copyright owner(s) are credited and that the original publication in this journal is cited, in accordance with accepted academic practice. No use, distribution or reproduction is permitted which does not comply with these terms. 